\title{
AC Followed by Pertuzumab/Trastuzumab/Docetaxel Regimen
}

National Cancer Institute

\section{Source}

National Cancer Institute. AC Followed by Pertuzumab/Trastuzumab/Docetaxel

Regimen. NCl Thesaurus. Code C138031.

A chemotherapy regimen consisting of cyclophosphamide and doxorubicin followed by pertuzumab, trastuzumab, and docetaxel, used as a neoadjuvant or adjuvant treatment for HER-2/neu-positive breast cancer. 